\title{
Electroless deposition of metallic silver from a choline chloride-based ionic liquid: a study using acoustic impedance spectroscopy, SEM and atomic force microscopy
}

\author{
Andrew P. Abbott, Satvinder Nandhra, Stella Postlethwaite, Emma L. Smith and \\ Karl S. Ryder*
}

Received 15th March 2007, Accepted 3rd May 2007

First published as an Advance Article on the web 22nd May 2007

DOI: $10.1039 / \mathbf{b 7 0 3 9 5 4 a}$

In this paper, we describe the first example of a sustained galvanic coating deposited on a surface from a non-aqueous liquid. We present the surface characterization of electroless silver deposits on copper substrates from a solution of $\mathrm{Ag}^{+}$ions in an ionic liquid based on a choline chloride $(\mathrm{ChCl})$ eutectic. Through a study of these deposits and the mechanism of formation using acoustic impedance spectroscopy (QCM), probe microscopy (AFM) and electron microscopy (SEM/EDX), we demonstrate that sustained growth of the silver deposit is facilitated by the porous nature of the silver. This is in contrast to the dip-coating reaction of silver ions in aqueous media, where the reaction stops when surface coverage is reached. Electroless silver deposits of up to several microns have been obtained by dip coating in ionic liquids without the use of catalysts of strong inorganic acids.

\section{Introduction}

Here we describe the physical characterization of electroless silver deposits on copper substrates from a solution of $\mathrm{Ag}^{+}$ ions in an ionic liquid solvent based on a choline chloride $(\mathrm{ChCl})$ eutectic.

The electroless deposition of silver metal onto copper substrates is an important industrial process that is used primarily by the manufacturers of printed circuit boards to prevent degradation of exposed copper surfaces (conduction tracks) during the time that elapses between the manufacture of the circuit board and the assembly procedure incorporating the board or component into a finished device. Typically this can be up to several months. This method of protection for the copper surfaces is very effective despite the fact that silver is thermodynamically much more susceptible to aerobic oxidation. This is because silver oxide and sulfide (formed as tarnish on silver surfaces), as well as the underlying silver metal, are very soluble in the tin- and tin/lead-based molten solders that are used to bond the circuit components to the copper tracks of the circuit board. On the contrary, copper oxides are poorly soluble in the molten solder such that aerobic oxidation of the copper surface prior to soldering inhibits interfacial wetting at the copper/solder interface and prevents bonding of the component. In a practical device, this results in failure of the joint either through poor conductivity or because of low mechanical stress tolerance. Solder fluxes are often used to mitigate this problem by preventing aerobic oxidation of copper surfaces, but this approach is not always successful or practical. Commercial electroless silver processes typically use an aqueous $\mathrm{AgNO}_{3}$ solution in the presence of $\mathrm{HNO}_{3}$. In addition to the

Chemistry Department, University of Leicester, Leicester, UK LE1

7RH.E-mail: k.s.ryder@le.ac.uk; Fax: + 44 (0)116 2523789 social and environmental concerns that surround the use of strong inorganic acids, here the use of $\mathrm{HNO}_{3}$, whilst necessary to the process, has an additional detrimental effect because there is competitive etching of the copper tracks during silver plating. This can be a serious problem for the electronics industry, particularly as integration densities continue to increase and consequently feature sizes become smaller. Many component failures arise because of copper etching (by the $\mathrm{HNO}_{3}$ ) before the surfaces are silver plated. In addition, the aqueous process requires the use of a colloidal catalyst (usually palladium metal) to sustain silver plating from beyond a few nanometres up to $1-5 \mu \mathrm{m} \dagger$ in thickness. ${ }^{1,2}$

Ionic Liquids (IL) have recently received a great deal of attention in the literature as alternative solvents and electrolytes for a number of electrochemical processes. ${ }^{3,4}$ These processes range in scope from fundamental academic investigations, for example into the structure of the solid/liquid and liquid/liquid interfaces ${ }^{5}$ or into the mechanisms of ion diffusion, to industrial processes where the IL has the potential to replace conventional noxious aqueous media. ${ }^{6}$ We have recently described the applications of a relatively new class of IL based on eutectic mixtures of choline chloride with a hydrogen bond donor species. ${ }^{7-9}$ These liquids can be used for the deposition of a range of metal coatings including $\mathrm{Zn}, \mathrm{Cr}, \mathrm{Sn}$, $\mathrm{Cu}, \mathrm{Ag}$, at high current efficiency and also for metal dissolution processes such as electropolishing. ${ }^{10,11}$ In many cases, the use of IL solvents overcomes the need for strong inorganic acids (e.g., chromic acid, $\mathrm{HF}, \mathrm{H}_{2} \mathrm{PO}_{4}, \mathrm{H}_{2} \mathrm{SO}_{4}$ ) and highly toxic salts (e.g., cyanide). Here, we describe the deposition and morphology of metallic electroless silver coatings from

$\dagger$ Dip coating of $\mathrm{Ag}$ onto $\mathrm{Cu}$ is spontaneous in aqueous solution but growth of the Ag deposit in the absence of a catalyst is not sustained beyond surface coverage. 
solution in an IL based on a choline chloride (ChCl), ethylene glycol (EG), $\mathrm{ChCl}: 2 \mathrm{EG}$, eutectic mix. Crucially, the nature of the morphology of silver deposition facilitates sustained growth of silver coatings. This is in contrast to the aqueous dip-coating reaction. We use acoustic impedance spectroscopy performed with a Quartz Crystal Microbalance (QCM) together with AFM and SEM to probe the mechanism of deposition and the structure of the silver deposits. Electroless silver deposits of up to several microns have been obtained by dip coating in ionic liquids without the use of catalysts of strong inorganic acids.

\section{Experimental}

Choline chloride ( $\mathrm{ChCl}$ ) (Aldrich 99\%) was recrystallised from absolute ethanol, filtered and dried under vacuum. Ethylene glycol (EG) (Aldrich 99+\%) was used as received. The mixture was formed by stirring the two components in the ratio $1 \mathrm{ChCl}: 2 \mathrm{EG}$, at $75^{\circ} \mathrm{C}$ until a homogeneous colourless liquid was formed. Voltammetry was carried out using an Autolab PGSTAT12 potentiostat controlled using GPES software. A three-electrode system consisting of a platinum disc working electrode ( $0.5 \mathrm{~mm}$ radius), sealed in glass, a platinum counter electrode and a silver wire reference electrode were used.

Acoustic impedance QCM experiments were carried out using electrodes consisting of thin $\mathrm{Au}$ films (no $\mathrm{Ti}$ or $\mathrm{Cr}$ binding layer was used) evaporated on $10 \mathrm{MHz}$ quartz crystals (International Crystal Manufacturing Co., Oklahoma City, USA); the surface finish of these crystals was either polished or unpolished. The piezoelectric active electrode area was $0.23 \mathrm{~cm}^{2}$. Crystal impedance spectra were recorded using a Hewlett Packard HP8751A network analyser, connected to a HP87512A transmission/reflection unit via $50 \Omega$ coaxial cable such that the centre of the spectrum was near the centre of resonance, $f_{0},(10 \mathrm{MHz})$ with a typical sweep width of 20 $200 \mathrm{kHz}$ depending on the interface. Measured data were fitted to a Lorentzian equivalent circuit model (eqn (1)) incorporating the in-phase impedance, inductance and centre frequency with a simple iterative difference method using Microsoft Excel (fitting procedures, etc. are discussed in more detail elsewhere $\left.{ }^{12-14}\right)$. In order to collect the admittance spectra with greater temporal resolution, network analyser data acquisition was controlled by a computer running HP VEE. This program was capable of recording admittance spectra every 3-4 s, which permitted the investigation under dynamic potential control. The crystal was fixed into the simple cell so that one face of the crystal was exposed to the solution. The other face was exposed to air.

$$
U(f)=a+\frac{R}{R^{2}+16 \pi^{2} L^{2}\left(f-f_{0}\right)^{2}}
$$

where $U(f) / \Omega^{-1}$ is the measured admittance curve as a function of applied frequency, $f / \mathrm{Hz}$ (and $U(f)=1 / Z(f)), R / \Omega$ is the real component of the impedance $(Z), L /$ Henry is the inductance, $f_{0} / \mathrm{Hz}$ is the centre frequency and $a / \Omega^{-1}$ is the baseline offset. The latter was used during fitting to compensate for variations in the static calibration of the network analyser.
AFM images were acquired using a Digital Instruments (DI) Nanoscope IV, Dimension 3100 instrument using either resonant (tapping) or contact mode (software version 6.12).

Surface analysis with SEM and elemental analysis using EDX were carried out under vacuum using a Philips XL30 ESEM instrument with an accelerator voltage of between $15-20 \mathrm{kV}$, giving a typical beam current of $c a .120 \mu \mathrm{A}$. Gold sputter coating was not required in most cases because of the high intrinsic conductivity.

Copper substrates for silver deposition were typically $10 \times$ $40 \mathrm{~mm}$ and $2 \mathrm{~mm}$ in thickness. Prior to silver deposition experiments, the substrates were first etched using $5 \%$ aqueous $\mathrm{HCl}$ then rinsed with distilled $\mathrm{H}_{2} \mathrm{O}$, dried and polished mechanically (using a rotary buffing wheel). The copper substrates were then cleaned using dichloromethane and kept under acetone until immediately prior to use.

All deposition experiments were carried out at room temperature (typically $20-23{ }^{\circ} \mathrm{C}$ ) unless otherwise specifically stated. Regions of the samples were masked off from deposition using acrylic resin dissolved in ethyl acetate or acetone.

Electrolytic deposition of copper onto gold QCM crystal surfaces was accomplished in a $0.1 \mathrm{M}$ solution of $\mathrm{CuSO}_{4}$ in water using potential step chronoamperometry with an applied potential of $-0.3 \mathrm{~V}$ (versus $\mathrm{Ag}$ wire pseudo reference). The time scale was varied according to the desired deposition charge (typically $20-50 \mathrm{~s}$ ).

\section{Results and discussion}

Deep eutectic solvents (also referred to here as ionic liquids) formulated from stoichiometric mixtures of choline chloride and either ethylene glycol (EG) or urea are especially good solvents for a range of metal salts and metal oxides that are not otherwise soluble in aqueous media. ${ }^{7-9} \mathrm{~A}$ range of silver salts including $\mathrm{AgNO}_{3}, \mathrm{AgCl}, \mathrm{AgSO}_{4}$ and $\mathrm{Ag}\left(\mathrm{CH}_{3} \mathrm{COO}\right)$ are soluble in both $\mathrm{ChCl}: 2 \mathrm{EG}-$ and $\mathrm{ChCl}: 2$ Urea-based ionic liquids. Cyclic voltammetry of these silver salts in each type of IL are indistinguishable, however, (with the mid-point potential between the deposition and stripping occurring at $0 \mathrm{~V}$ with respect to a silver wire reference) suggesting that the $\mathrm{Ag}^{+}$ speciation is dominated by the nature and formulation of the IL rather than by the counterion of the salt. This is most probably because of the very high concentration of chloride ions in the IL (typically $4.8 \mathrm{~mol} \mathrm{~L}^{-1}$ ) in comparison to the silver salt concentration (typically $10 \mathrm{mmol} \mathrm{L}^{-1}$ ). Fig. 1 shows the cyclic voltammetry of a mixture of $\mathrm{CuCl}_{2}$ and $\mathrm{AgCl}$ in the EG-based liquid. Here, three distinct redox processes are clearly visible, Fig. 1. The first of these, Fig. 1(a), $E=$ $-400 \mathrm{mV}$, shows the deposition and stripping response of $\mathrm{Cu}$ corresponding to the reduction of $\mathrm{Cu}^{+}$. Similarly, the process shown in Fig. 1(b), $E=0 \mathrm{~V}$, corresponds to the deposition and stripping response of Ag. The process labelled Fig. 1(c), $E=+425 \mathrm{mV}$, corresponds to the reversible, solution, $\mathrm{Cu}^{2+/+}$ couple. These data show clearly that $\mathrm{Ag}^{+}$ion reduction is thermodynamically favoured upon immersing $\mathrm{Cu}$ metal into a solution of $\mathrm{AgCl}$ in EG-based IL. Additionally, it is also clear from the thermodynamics (redox potentials) that the consequential dissolution product accompanying silver deposition is $\mathrm{Cu}^{+}$, rather than $\mathrm{Cu}^{2+}$, according to Scheme 1 


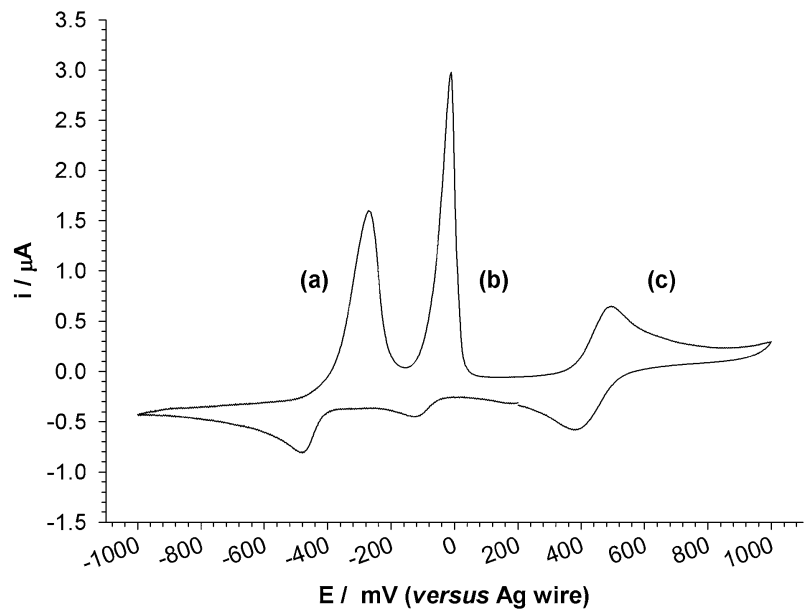

Fig. 1 Cyclic voltammogram of a mixture of $\mathrm{CuCl}_{2}$ and $\mathrm{AgCl}$ in a $\mathrm{ChCl}$ : 2EG IL at a Pt disc $(0.5 \mathrm{~mm}$ dia. $)$ working electrode and a potential scan rate of $\nu=20 \mathrm{mV} \mathrm{s}^{-1}$. Process (a) $\mathrm{Cu}^{+}$reduction and stripping, (b) $\mathrm{Ag}^{+}$reduction and stripping, (c) $\mathrm{Cu}^{2+/+}$ redox couple. Potential measured versus a silver wire reference electrode.

$$
\mathrm{Ag}_{(I L)}^{+}+\mathrm{Cu}_{(S)} \rightleftharpoons \mathrm{Cu}_{(I L)}^{+}+\mathrm{Ag}_{(S)}
$$

(the state designation "IL" is used to signify the solvated ion in the IL). Interestingly, both $\mathrm{Cu}(\mathrm{I})$ and $\mathrm{Cu}(\mathrm{II})$ salts are seen to be stable in the $\mathrm{ChCl}: 2 \mathrm{EG} \mathrm{IL}$ and there is no evidence of $\mathrm{Cu}(\mathrm{I})$ disproportionation seen in aqueous solution. However, FAB mass spectrometry analysis of $\mathrm{Cu}(\mathrm{I}), \mathrm{Cu}(\mathrm{II})$, and $\mathrm{Ag}(\mathrm{I})$ salts in this liquid have identified $\left[\mathrm{CuCl}_{2}\right]^{-},\left[\mathrm{CuCl}_{3}\right]^{-}$and $\left[\mathrm{AgCl}_{2}\right]^{-}$ complex anions, respectively. Hence, the relatively high concentration of chloride ions in the ionic liquid explains why disproportionation of $\mathrm{Cu}(\mathrm{I})$ is not observed here.

\section{Electroless Ag deposition from $\mathrm{ChCl}$ : 2EG}

In a typical procedure, a sample of mechanically polished (mirror finish) copper metal was immersed in $\mathrm{ChCl}: 2 \mathrm{EG}$ ionic liquid containing $10 \mathrm{mmol} \mathrm{L}^{-1} \mathrm{AgCl}$ for a period of $5 \mathrm{~min}$. The sample was then removed and sequentially rinsed with fresh IL, propanol, and methanol. A region of the sample surface had been masked by painting with a solution of acrylic resin (dissolved in ethyl acetate), which was subsequently removed using acetone to reveal a clear boundary edge between the silver deposit and the uncoated copper substrate. Such samples were then examined under the SEM. The resulting SEM image, presented in Fig. 2(a), shows the two regions of the sample prepared in the manner described above. The uncoated $\mathrm{Cu}$ substrate is seen at the bottom of the image and the silver deposit at the top. The horizontal boundary separating the two regions is clearly visible approximately two thirds of the way down the image. The copper substrate is relatively smooth, showing some mechanical tool marks from the polishing procedure and pits and scratch marks. The morphology of the silver deposit is qualitatively very rough in comparison, the surface features of the substrate are no longer visible in this region. Closer inspection of the silver deposit at higher magnification, Fig. 2(b), shows that the silver deposit has a nodular structure with randomly distributed voids that are of similar magnitude to the nodules. Spectroscopic analysis (EDX) of the two surfaces showed only the presence of $\mathrm{Cu}$ and $\mathrm{Ag}$ emission peaks, respectively. Dip coatings of silver deposits were also obtained on copper substrates from solutions of other silver salts: $\mathrm{AgNO}_{3}, \mathrm{AgSO}_{4}$ and $\mathrm{Ag}\left(\mathrm{CH}_{3} \mathrm{COO}\right)$. Despite the similarity in the voltammetric responses of these silver salts, the dip-coatings of silver varied significantly, although not systematically, in qualitative appearance. In order to acquire quantitative data on thickness and morphology of the deposits, samples were examined using probe microscopy.

\section{Atomic force microscopy}

Copper samples plated with electroless silver deposits were examined using scanning AFM in either resonant (tapping) or contact mode. A typical data set is shown in Fig. 3. Fig. 3(a) shows the $x y z$ projection of a silver coated sample with the boundary between coated (right) and uncoated $\mathrm{Cu}(l e f t)$ running diagonally across the image (left-right). The 2D projection of this image is shown as Fig. 3(b); here, the silver coated region is seen in the bottom left diagonal segment. Both of these two representations show clearly that the surface roughness of the silver deposit is orders of magnitude greater than that of the substrate. Height data extracted from the line joining bottom-left and top-right hand corners of the image shown in Fig. 3(b) are presented in Fig. 3(c). These data show quantitatively the magnitude of surface roughness over the silver coating, but also clearly show the thickness of the silver layer, having a mean thickness of $c a .500 \mathrm{~nm}$ above the mean
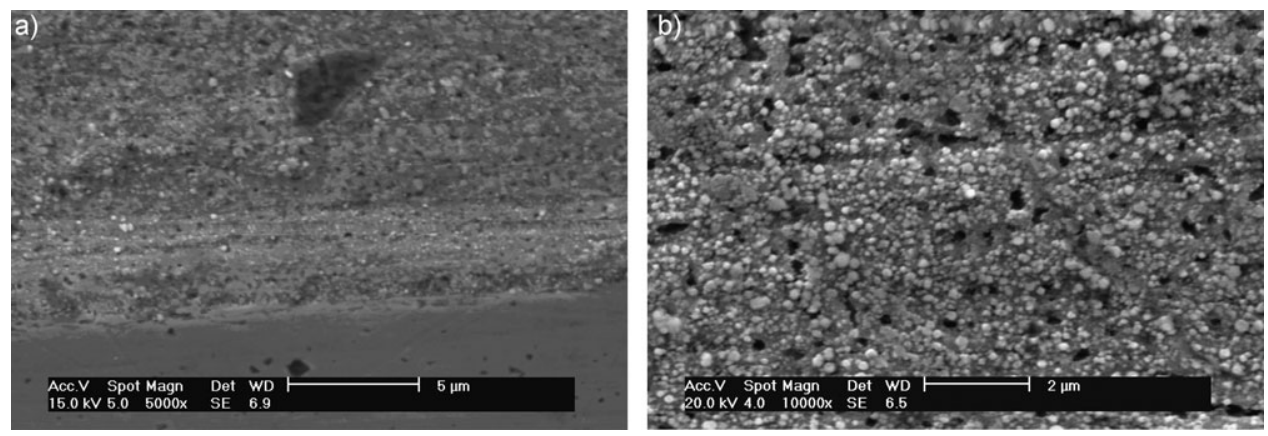

Fig. 2 Scanning electron micrograph of a polished $\mathrm{Cu}$ sample exposed to $\mathrm{AgCl}$ solution of $\mathrm{ChCl}: 2 \mathrm{EG}$ IL for 5 min: (a) image showing both uncoated $\mathrm{Cu}$ substrate and $\mathrm{Ag}$ coated regions, (b) higher magnification image showing the $\mathrm{Ag}$ coated region only (from image (a)). 

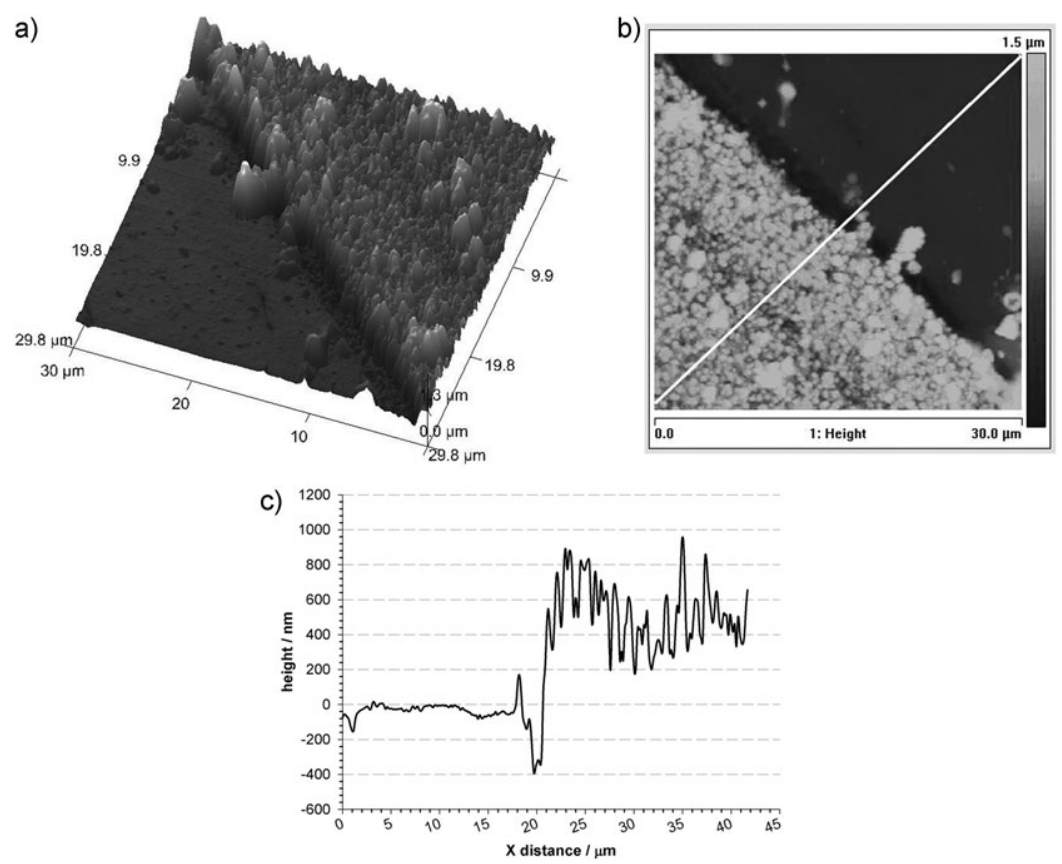

Fig. 3 AFM image (resonant mode) of a Ag plated Cu sample (SEM shown in Fig. 2); (a) $x y z$ surface projection, (b) height contrast image of the same data, (c) height/distance data for the diagonal line trace sown in (b).

plane defined by the copper substratet. Also present in these data (Fig. 3(c)) is evidence of a narrow depression at the boundary between the copper substrate and the silver coating. This depression, approximately $2 \mu \mathrm{m}$ wide and $400 \mathrm{~nm}$ deep, extends the length of the boundary forming a narrow trough that seems to have been etched from the substrate. Control experiments were performed in which the copper substrate, coated with acrylic mask, was soaked in neat IL (in the absence of $\mathrm{Ag}^{+}$ion). Examination of the sample under AFM, after subsequent rinsing and removal of the mask, showed no boundary features. Consequently, the presence of the "trough" between the copper and silver deposit is not an artefact of the experimental procedure. We speculate that copper must be removed from the substrate to facilitate silver deposition and that the level at the bottom of this depression defines the total degree of etching over the plane of the copper substrate. Hence, the total thickness of silver could be estimated as ca. $900 \mathrm{~nm}$.

Surface roughness is often expressed as a ratio of the measured topographical surface area, $A_{\mathrm{M}}$, calculated from the height data and the projected geometric sample area (i.e. footprint), $A_{\mathrm{P}}$, (here this is $25 \mu \mathrm{m}^{2}$ ). Surface roughness, $R$, is then expressed as a percentage according to eqn (2).

$$
R=\left(\frac{A_{m}}{A_{\mathrm{P}}}-1\right) \times 100 \%
$$

Using this method, the silver plated region has an average roughness of $45 \%$ compared to a value of $1 \%$ for the uncoated substrate. This experiment was repeated using a lower concentration of $\mathrm{Ag}^{+}$ions $\left(1 \mathrm{mmol} \mathrm{L}^{-1}\right)$ in order to slow the rate

$\ddagger$ No real-time plane fitting was applied during the acquisition of the AFM images; however, plane fitting was subsequently performed on the raw data using the uncoated $\mathrm{Cu}$ substrate as the reference plane. of deposition. Subsequently, the surface roughness was measured as a function of deposition time. In addition, using a fixed deposition time of $5 \mathrm{~min}$, the surface roughness was measured on a further set of samples over a range of temperatures. The data obtained are summarised in Tables 1 and 2. From these data, it is clear that during the deposition reaction surface roughness increases and that raising the temperature also increases the surface roughness (rate of deposition) of the silver deposit.

Quantitative and qualitative differences were observed between samples of silver deposit obtained from various silver salts, however, no trends were observed. Dip-coatings from $\mathrm{AgCl}$ gave the most consistent results and so we have focussed our investigation on the formation of silver dip-coatings from $\mathrm{AgCl}$ solution.

One striking feature of the dip-coated silver deposit shown in Fig. 3 is the overall thickness ( $c a .0 .5 \mu \mathrm{m}$ at the mask boundary). This is much thicker than might be anticipated on the basis of a conventional (aqueous) dip-coating mechanism. The mechanism of aqueous (dip-coating) deposition is driven by the thermodynamics (i.e. difference in redox potential) in just the same way but deposition stops when the surface is covered with silver, since the new silver surface masks the copper from the aqueous $\mathrm{Ag}^{+}$and prevents dissolution of the

Table 1 Surface roughness measurements as a function of time for polished copper substrates exposed to the $\mathrm{AgCl}$ solution in $1 \mathrm{ChCl}$ : 2EG

\begin{tabular}{lll}
$\begin{array}{l}\text { Sample deposition } \\
\text { time/min }\end{array}$ & $\begin{array}{l}\text { Surface area ratio \% } \\
\text { Ag deposit }\end{array}$ & $\begin{array}{l}\text { Surface area ratio \% Cu } \\
\text { substrate }\end{array}$ \\
\hline 5 & 1.5 & 0.25 \\
10 & 3.0 & 0.5 \\
15 & 6.0 & 0.3 \\
\hline
\end{tabular}


Table 2 Surface roughness measurements as a function of temperature for polished copper substrates exposed to the $\mathrm{AgCl}$ solution in $1 \mathrm{ChCl}: 2 \mathrm{EG}$ for a fixed period of time $(5 \mathrm{~min})$

\begin{tabular}{lll}
\hline $\begin{array}{l}\text { Sample deposition } \\
\text { temperature } /{ }^{\circ} \mathrm{C}\end{array}$ & $\begin{array}{l}\text { Surface area } \\
\text { ratio } \% \mathrm{Ag} \text { deposit }\end{array}$ & $\begin{array}{l}\text { Surface area } \\
\text { ratio } \% \mathrm{Cu} \text { substrate }\end{array}$ \\
\hline 30 & 1.0 & 0.2 \\
50 & 9.0 & 0.8 \\
70 & 12.5 & 0.5 \\
\hline
\end{tabular}

copper ions from the underlying metal. Hence, it is clear from our results that the mechanism of electroless deposition of silver from $\mathrm{ChCl}: 2 \mathrm{EG}$ IL is very different to that in aqueous solution.

\section{Acoustic impedance spectroscopy using QCM}

In order to investigate the rate of deposition of silver during the electroless process we have used a resonant QCM as a mass probe. Most commercial QCM instruments use a derivative technique that locks and tracks the peak frequency of crystal resonance as a function of time. This technique does not work well with IL solvents because the relatively high viscosity attenuates the crystal resonance. Using acoustic impedance spectroscopy, we acquire the full resonance spectrum of the in situ mass-loaded crystal at any point in time. Subsequent curve fitting yields the peak frequency and the real component of the impedance for the crystal circuit. This is particularly useful in analysis because the electrical impedance of the crystal circuit is coupled to the mechanical losses that are sustained at the crystal/liquid interface. These losses are, in turn, characteristic of that interface, giving potentially useful quantitative information about surface roughness and density. In addition, a direct empirical measure of these mechanical losses is available from the $Q$ factor, $Q$, of the admittance resonance curve. The $Q$ factor of a resonance curve is a measure of the broadness of resonance given by eqn (3),

$$
Q=f_{0} / w
$$

where $f_{0}$ is the frequency value at the centre of resonance and $w$ is the Full Width of the peak at Half Height (FWHH). Such resonance curves are conventionally presented as admittance, $U /$ Siemens, frequency plots where admittance is the reciprocal of impedance, $Z / \Omega$.

The impedance QCM experiments were carried out using either a polished (flat mirror) or unpolished "frosted" finish crystal in a simple cell such that the working face of the crystal was bonded to a circular opening in the base of a quickfit vessel similar to that described elsewhere. ${ }^{12-14}$ In a typical experiment, the admittance spectrum for a dry unpolished crystal was first acquired prior to any surface treatment. The gold surface of the crystal was then coated with an electrolytic deposit of copper metal. This was most easily achieved using potential step chronoamperometry in a conventional three electrode electrochemical cell, using aqueous $\mathrm{CuSO}_{4}$ solution (see Experimental section). During electrolytic copper deposition the current was monitored as a function of time to enable the calculation of Faradaic charge. $\S$ The $\mathrm{CuSO}_{4}$ solution was

§ The applied potential was kept sufficiently small so as to maintain a high current efficiency for copper deposition. then removed from the cell, the crystal was rinsed with fresh water, dried and the admittance spectrum of the dry copper coated crystal recorded. Subsequently, a $\mathrm{AgCl}$ solution in $\mathrm{ChCl}$ : 2EG IL was added to the cell and the admittance spectrum of the crystal (in contact with the liquid) was continually recorded using a repetitive scan. At the end of a time period after which the experiment was judged to be complete the IL solution was removed from the cell, the crystal was then rinsed and dried and the admittance spectrum of the dry silver coated crystal was recorded. All of the recorded spectra were then numerically processed (see Experimental section) to yield the peak frequency and $Q$ factor.

\section{Frequency trends}

A complete data set of 30 admittance curves recorded during a silver deposition experiment is shown in Fig. 4(a). At time, $t=0$, the admittance curve has a peak value $U_{\text {peak }}=1.0 \mathrm{mS}$ and a $Q$ factor $=401$ (measured from the data), which are both quite small and characteristic of an attenuated resonance when compared with corresponding values for the dry coppercoated crystal; $U_{\text {peak }}=20 \mathrm{mS}$ and $Q=24160$. These observations are consistent with the intrinsic mechanical losses associated with the interface between the IL and the crystal. Each of the 30 spectra were numerically fitted; the resultant values of frequency shift, $\Delta f_{0} \|$, are plotted as a function of time in Fig. 4(b). Here, a downward frequency trend is visible corresponding to an increase in mass of the crystal. This is consistent with the replacement of $\mathrm{Cu}$ with $\mathrm{Ag}$. The trend continues for approximately $12 \mathrm{~min}$ and then the resonant frequency stabilises at around $-10 \mathrm{kHz}$. No further change was observed.** It is clear from Fig. 4(a) that although the position of the resonance peak has moved to lower frequency, the shape of the resonant curve is qualitatively unchanged. This is confirmed by $Q$ factor measurements at the beginning and end of the deposition, where $Q(t=0)=401$ and $Q(t=$ $16 \mathrm{~min})=378$. Hence, the broadness of the resonance has changed by less than $7 \%$ during deposition.

This experiment was repeated several times using the same crystal (following cleaning of the crystal surface by removal of the silver/copper deposit), each time increasing the total mass of $\mathrm{Cu}$ deposited. Another sample data set is shown in Fig. 4(c) and the corresponding $\Delta f_{0}(t)$ data are presented as Fig. 4(d). In this case, the total Faradaic charge for copper deposition was $0.23 \mathrm{C}$, whereas that in the previous example, Fig. 4(a-b), was $0.066 \mathrm{C}$. The data set Fig. 4(c) is striking in comparison to the previous case for two reasons; (i) the time scale is very much longer, (ii) the peak frequency of the admittance curve has both shifted to a lower frequency and has become acutely attenuated. This latter point is important and will be the focus of discussion in a following section. Fig. 4(d) (i) shows the

\footnotetext{
- The experimental time scale for the deposition process was estimated by visual monitoring of the peak position of the admittance curve. When this was no longer seen to be moving as a function of time the experiment was judged to be complete.

$\|$ Here, $\Delta f_{0}=f_{0}(t=0)-f_{0}(t)$, where $f_{0}(t=0)$ is the peak frequency of the first spectrum at $t=0$, and $f_{0}(t)$ is the peak frequency of each subsequent spectrum at time, $t$.

** The slight frequency up turn at $14 \mathrm{~min}>t>12$ min may be due to surface reorganisation. We will publish separately on this issue.
} 

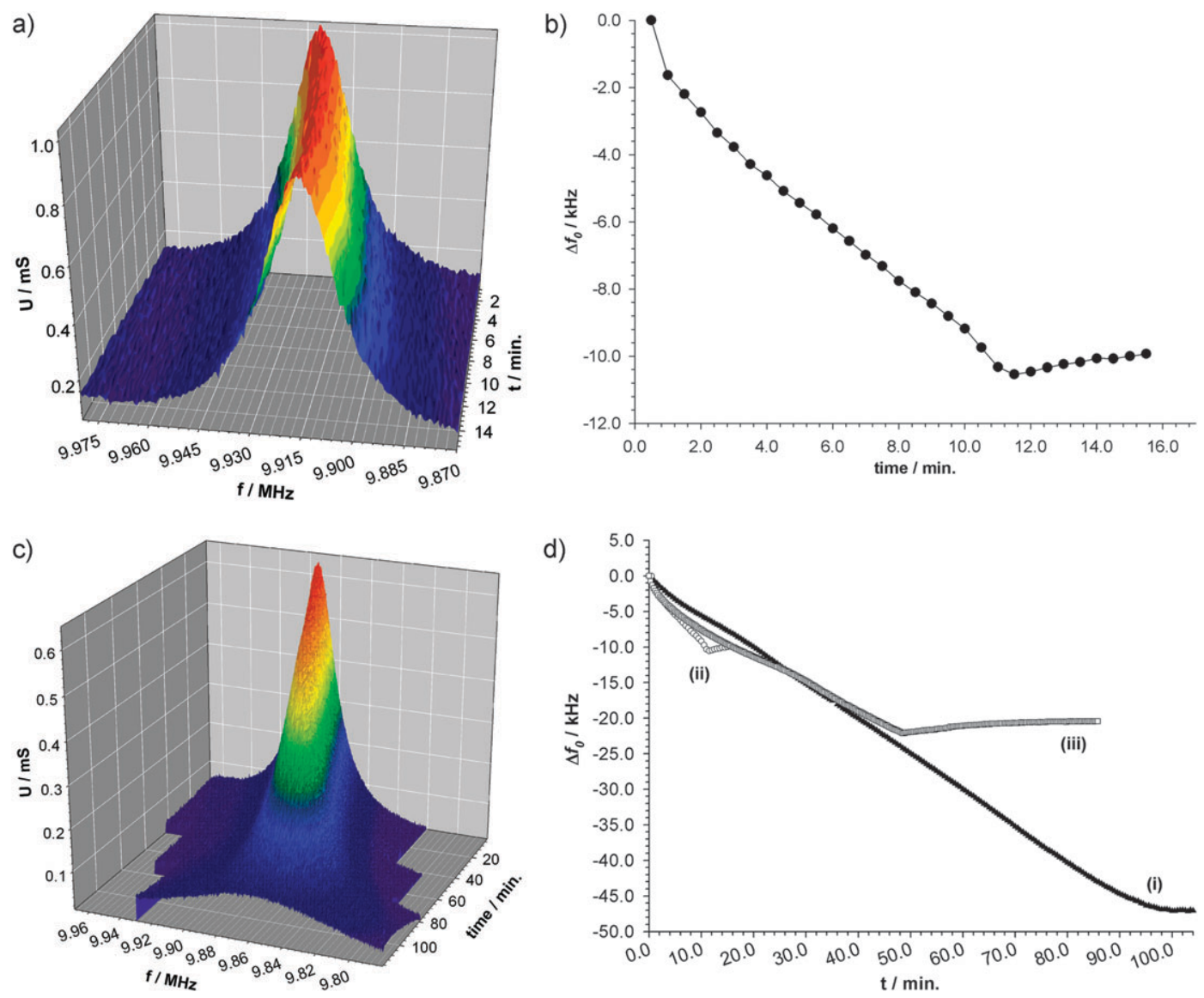

Fig. 4 Acoustic impedance data (QCM) for two silver deposition experiments. Experiment 1; copper deposition charge of $q_{\mathrm{Cu}}=0.066 \mathrm{C}$. (a) $U(f, t)$ data acquired (spectra recorded every $30 \mathrm{~s}$ ) during a silver deposition in $\mathrm{ChCl}: 2 \mathrm{EG}$ liquid, (b) peak difference, $\Delta f_{0}(t)$, data, obtained from numerical fitting of the spectra in (a). Experiment 2; copper deposition charge of $q_{\mathrm{Cu}}=0.23 \mathrm{C}$. (c) $U(f, t)$ data acquired (spectra recorded every $4 \mathrm{~s}$ ) during a silver deposition in $\mathrm{ChCl}: 2 \mathrm{EG}$ liquid, (d) $\Delta f_{0}(t)$ data, obtained from numerical fitting of (i) the spectra in Fig. 4(c), (ii) $\Delta f_{0}(t)$ data from Experiment 1, (b), presented for clarity and comparison, (iii) $\Delta f_{0}(t)$ data from a third experiment; $q_{\mathrm{Cu}}=0.107 \mathrm{C}$.

corresponding $\Delta f_{0}(t)$ data extracted from the spectra. Once again, the frequency data show a downward trend corresponding to increasing mass, culminating in a frequency change of $-47 \mathrm{kHz}$ after a time of $c a .100 \mathrm{~min}$. (The data plotted as Fig. 4(d) (ii) are taken from Fig. 4(b) and represented on the same scale for comparison.) The data for a third similar experiment are shown in Fig. 4(d) (iii); here, the initial Faradaic charge for the copper deposit was at an intermediate value of $0.107 \mathrm{C}$; the rate of frequency change was close to zero after $49 \mathrm{~min}$, resulting in a value of $\Delta f_{0}=-22 \mathrm{kHz}$. Clearly the variation in deposition time scale and the final value of $\Delta f_{0}$ correlate with the mass of silver deposited and with mass of copper initially electroplated onto the crystal. These data, together with the results of several other similar experiments, are summarised in Fig. 5, where the silver deposition time (i.e. time taken for the frequency response to level out) is plotted against the Faradic charge for the copper substrate layer. These results show clearly that deposition time is a function of the amount of copper present, and that consequently we have observed sustained deposition of electroless silver. The data presented in Fig. 5 also comprise experiments performed at both polished and unpolished crystal surfaces. The correlation appears not to be sensitive to the morphology of the underlying electrode substrate.
The strong attenuation of the resonance curves observed in the $U(f, t)$ data of several experiments, particularly at long time scales, see Fig. 4(c), indicates mechanical viscoelastic loss at the crystal liquid interface. These losses preclude direct conversion of the frequency change, $\Delta f_{0}$, into equivalent mass, i.e., the Sauerbrey equation can not be used here. However, this information can be obtained directly from the dry crystal measurements before and after the experiment.

Spectra for another sample experiment are shown in Fig. 6. These resonances are very sharp and well defined (evidenced by the large values of $Q$ ). In addition, the peak intensities are very similar indicating that in each instance the crystal is behaving as a rigid resonator and that progressive losses after each deposition $(\mathrm{Cu}$ then $\mathrm{Ag})$ are small. Under these circumstances the shift in peak frequency can be used to calculate change in mass at the crystal according to the Sauerbrey equation. ${ }^{15}$ The peak frequency value, $f_{0}$, for the gold only coated crystal was taken as a reference zero value, consequently the peak of the resonance curve for the copper coated crystal occurs at a frequency of $f_{0}=-28.8 \mathrm{kHz}$ and that of the silver coated crystal occurs at $f_{0}=-45.0 \mathrm{kHz}$. Calculation of equivalent masses yields a value of $500 \mathrm{nmol}$ of $\mathrm{Cu}$ metal electrolytically deposited on the gold surface. (Integration of the current-time trace during copper deposition to give 


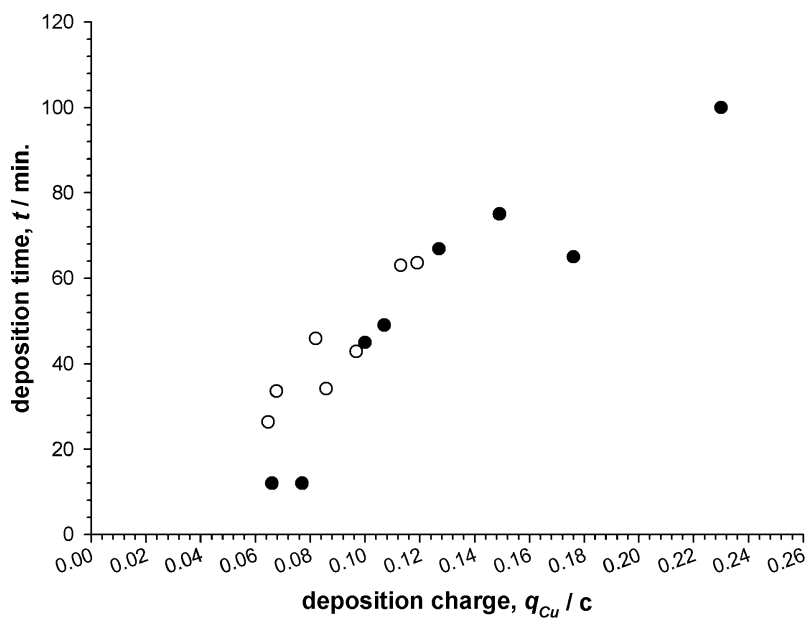

Fig. 5 Plot of silver deposition time (i.e., time for rate frequency change with time to level out $\left(\mathrm{d}\left(\Delta f_{0}\right) / \mathrm{d} t \approx 0\right)$ versus quantity of copper metal deposited on the crystal. The latter is expressed as the charged passed, $q_{\mathrm{Cu}}$, during electrolytic deposition; $(\bigcirc)$ Polished crystal surface, $(\bullet)$ unpolished crystal.

Faradaic charge yielded a value of $550 \mathrm{nmol}$, giving a current efficiency of $c a .91 \%$.) The corresponding value for silver is $459 \mathrm{nmol}$. This shows that within an experimental error (typically 5-10\%), all of the copper deposited on the crystal is replaced by silver and that the stoichiometry of the reaction is consistent with Scheme 1. The balance of silver may have been lost from the friable interface during transfer.

\section{$Q$ factor trends}

During silver deposition experiments lasting over relatively long time scales $(t>30 \mathrm{~min})$ we have observed that the impedance curve becomes acutely attenuated towards the end

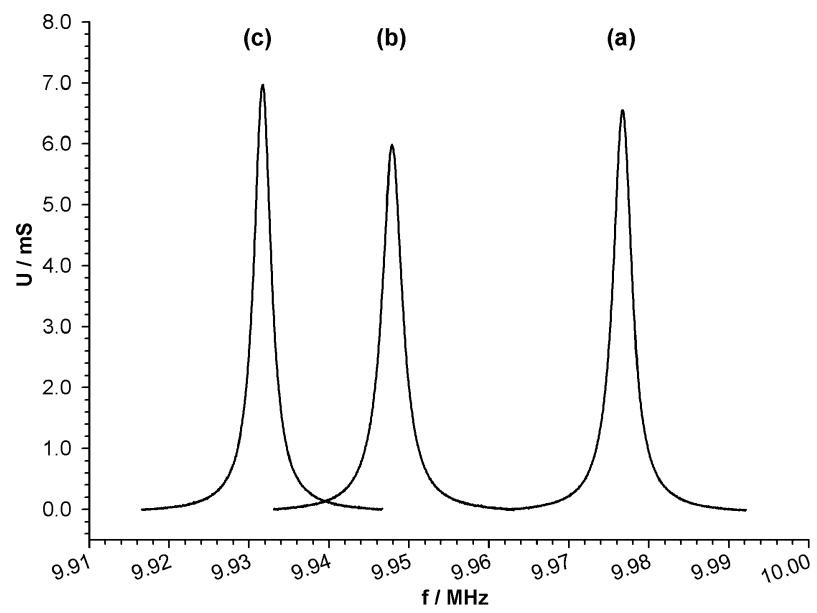

Fig. 6 Acoustic impedance spectra, $U(f)$, of (a) dry Au coated unpolished crystal untreated in air, $Q$ factor $=3528, f_{0}=9.97668$ $\mathrm{MHz}$, hence $\Delta f_{0}=0 \mathrm{~Hz}$ (i.e. this $f_{0}$ is defined as the reference frequency); (b) the same crystal (part (a)) $\mathrm{Cu}$ coated rinsed and dried, in air, $Q$ factor $=3237, f_{0}=9.94787 \mathrm{MHz}, \Delta f_{0}=-28.81 \mathrm{kHz}$ (c) the same crystal after completed silver deposition in $\mathrm{ChCl}: 2 \mathrm{EG}$ liquid, rinsed and dried in air, $Q$ factor $=3237, f_{0}=9.93166 \mathrm{MHz}, \Delta f_{0}=$ $-45.02 \mathrm{kHz}$. (Admittance, $U / \mathrm{mS}$, is the reciprocal of the measured impedance $Z / \Omega$.) of the deposition process. An example of this behaviour is shown in Fig. 4(c). The graph presented as Fig. 7(a) shows $Q$ factor data for this experiment as a function of elapsed time. Here, the $Q$ factor data measured directly from the spectra are shown (O) as well as the $Q$ factor data calculated from the best numerical fit ( - ). Good agreement between these two approaches is a semi-quantitative measure of the confidence in the numerical model. From these data, it is clear to see that during the first $40 \mathrm{~min}$ of the experiment the peak shape of the resonance curve remains fairly constant, with a $Q$ factor of between 240 and 320 . Subsequently, the $Q$ factor falls rapidly and continually until the rate of frequency change approaches zero after $120 \mathrm{~min}$. A similar trend is also evident when the $Q$ factor data are plotted as a function of frequency, $\Delta f_{0}$, Fig. 7(b). Early in the experiment, where frequency changes are small, $\Delta f_{0}<10 \mathrm{kHz}$, the $Q$ factor remains fairly constant. At later stages the increase in the magnitude of $\Delta f_{0}$ resulting from increased mass loading is accompanied by a decrease in $Q$ factor. This effect is most prominent towards the end of the deposition. These observations are consistent with increased viscoelastic losses at the crystal/liquid interface, particularly at high crystal mass loading. This is almost certainly caused by an increase in the surface roughness of the silver deposit. This
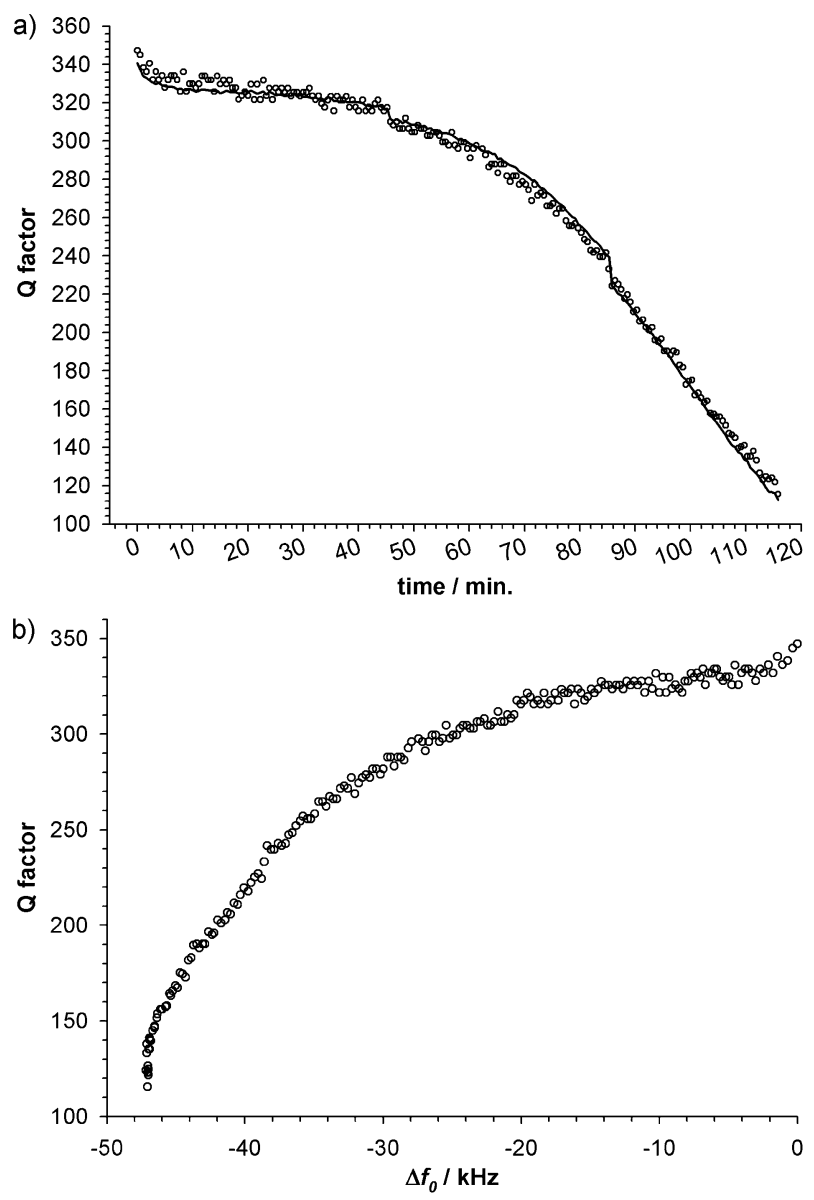

Fig. $7 Q$ factor data taken from Experiment 2, Fig. 5(c), plotted as a function of (a) time, the data points, $\bigcirc$, are measured directly from the spectra, the solid line represents the values of $Q$ calculated from the best fit; (b) peak frequency, $\Delta f_{0}$. 


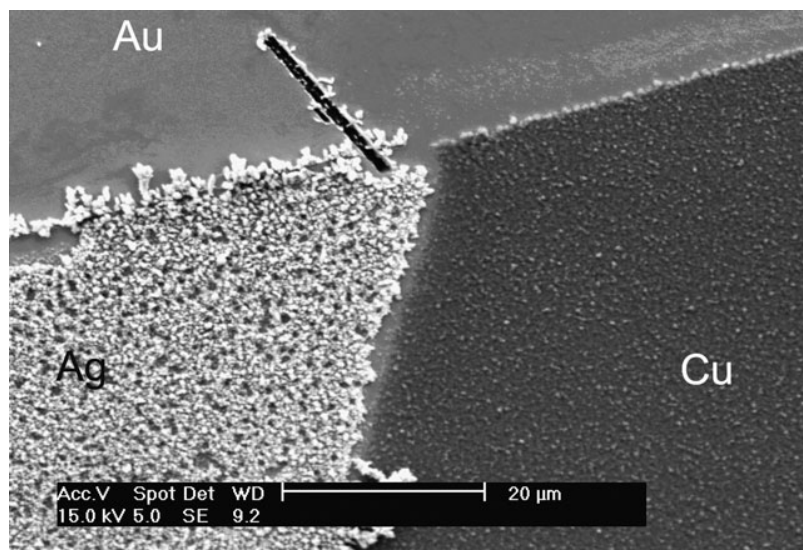

Fig. 8 SEM image of a polished quartz crystal showing the metal surfaces of the three stages of the silver coating experiment. These are marked on the image as the untreated Au region, the $\mathbf{C u}$ (electrolytic) coated surface, and the $\mathbf{A g}$ coated surface at the end of the experiment. (The linear feature at the top-centre of the image is a registration mark, i.e., scratch).

observation is confirmed by SEM analysis of one crystal surface where part of the gold substrate electrode and part of the copper coated surface were masked off prior to silver deposition. The SEM image presented as Fig. 8 shows a boundary region of this sample after the mask was removed at the end of the experiment. Here, three morphologies corresponding to the gold substrate (top), copper (bottom right) and silver (bottom left) are clearly visible. The surface roughness of the silver deposit is very much greater than that of the underlying copper that it has displaced. The surface roughness of the copper is also greater than that of the underlying substrate.

\section{Rate of silver deposition}

The rates of silver deposition $\mathrm{d}\left(\Delta f_{0}\right) / \mathrm{d} t$ for a range of experiments was measured directly from the $\Delta f_{0}(t)$ plots, see for example Fig. 4(b) and (d). In most cases these data show that a steady state growth phase was preceded by a much faster initial rate of deposition. These data are summarised in, Fig. 9, and incorporate data for deposition at both polished (circles) and unpolished (triangles) crystal surfaces. These data show a wide variation (close to a factor of 10) of initial rate but no correlation with the amount of copper deposited on the crystal. The variation of initial rate, however, seems most pronounced at the unpolished crystal surfaces. On the other hand the steady state rates show a variation of only around a factor of two (Fig. 9 insert). Again, no correlation is observed between the amount of copper present on the surface and the steady state rate. These results are consistent with a model in which there is an initial rapid nucleation of silver on the copper surface followed by a slower bulk growth mechanism that is likely to be limited by mass transport. Eventually, this mechanism is terminated when there is no more copper on the crystal surface.

The variation in initial rates can be rationalised with this model because the kinetics of nucleation are likely to be sensitive to small/localised effects such as surface roughness

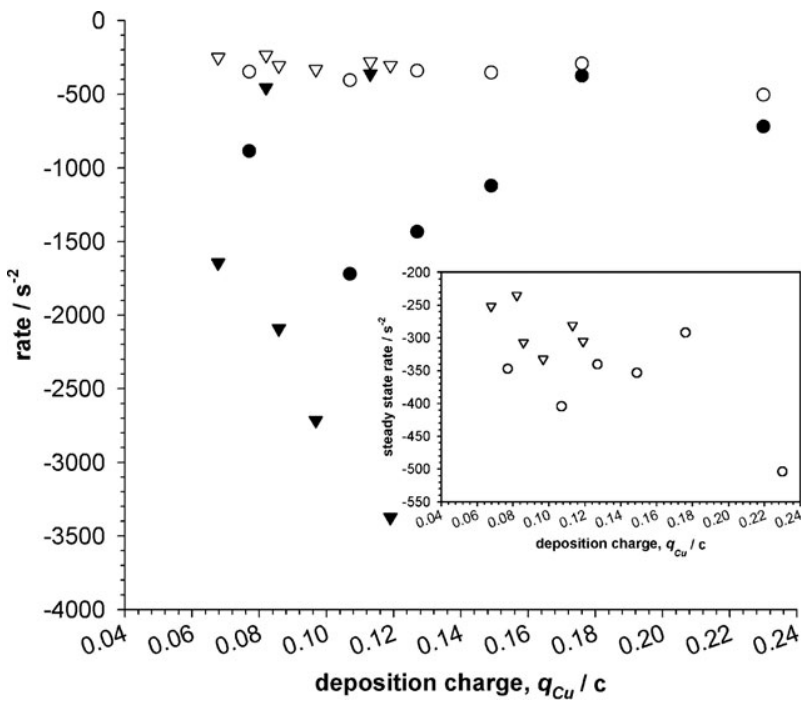

Fig. 9 Rates of silver deposition measured as, $\mathrm{d}\left(\Delta f_{0}\right) / \mathrm{d} t$, plotted as a function of the quantity of copper deposited on the crystal. The latter is expressed as the charge passed during electrolytic deposition. Initial rates: $(\boldsymbol{\nabla})$ polished crystal, $(\bullet)$ unpolished crystal surface. Initial rates steady state rates: $(\nabla)$ polished crystal, $(\bigcirc)$ unpolished crystal surface.

and concentration of nucleation sites; it follows that the rate of nucleation may vary in magnitude from surface to surface in each experiment. In particular, at a polished surface the concentration of nucleation sites may vary significantly from experiment to experiment whereas at an unpolished, relatively rough, surface the number of nucleation sites is already large.

The rough and porous nature of the silver deposit as evidenced by SEM, AFM and QCM suggests that the steady state rate of silver deposition may be limited by diffusion of the $\mathrm{Cu}^{+}$ions from the active surface of the crystal. This accounts for the relatively small variation in steady state rates. The observation that the silver deposition is sustained over long periods of time (where sufficient copper is present) suggests that this is possible through pores and channels in the silver deposit that are penetrated by the IL solution, facilitating both diffusion of $\mathrm{Ag}^{+}$in to the deposit and $\mathrm{Cu}^{+}$ions out. The latter model of a rough surface interpenetrated by liquid-filled pores and channels is also consistent with the viscoelastic losses (resonance damping) observed in the impedance QCM spectra of the crystal coated with thick silver deposits. ${ }^{16,17}$

\section{Conclusions}

Here, we have described the first example of a sustained galvanic coating of silver metal deposited on a copper surface from a non-aqueous IL. The reduction of silver ions in the IL is driven by the difference in redox potential with respect to that of the copper (I) ion. However, the Cu(I) complex is stable in the IL, showing no signs of disproportionation as is seen for the equivalent $\mathrm{Cu}(\mathrm{I})$ complexes in aqueous solution. This difference in behavior is almost certainly attributable to the high concentration of chloride ions in the IL. Electroless coatings of silver onto copper substrates from aqueous solutions are not sustainable without the use of catalysts because the thin silver coating quickly deactivates the copper surface. 
The silver ions in solution can no longer access the copper substrate and the copper ions have no diffusion pathway from the bulk metal substrate. Here, we have shown that during silver deposition in the IL that both silver ion access to the copper metal substrate and copper ion diffusion away from the substrate are maintained through pores and channels in the silver deposit. Hence, the non-catalytic deposition mechanism is maintained by a rough surface morphology. This has been observed using both SEM and AFM imaging techniques. In addition, QCM studies show two types of growth morphology: (i) a relatively dense, thin, phase where peak frequency changes are not accompanied by concomitant viscoelastic losses (i.e., the resonant peak shape remains constant), and (ii) a phase after sustained growth where acute viscoelastic damping of the crystal resonance is observed. The latter is consistent with a very rough surface interface interpenetrated by the ionic liquid solution. These observations may be of interest in the electroless plating industry, particularly the electronics manufacturing sector. Electroless silver deposits of up to several microns have been obtained by dip coating in IL without the use of catalysts of strong inorganic acids.

\section{Acknowledgements}

The authors would like to thank the EU under framework programme 6 for funding (project IONMET, http://www. ionmet.org/).

\section{References}

1 C. R. Shipley, US Patent 3,011,920, filed (1961).

2 S. S. Djokic, "Electroless Deposition of Metals and Alloys" in "Modern Aspects of Electrochemistry", ed. Brian E. Conway and Ralph E. White, Springer US, 2002, vol. 35, pp. 51-133.

3 J. Sun, D. R. MacFarlane and M. Forsyth, Electrochim. Acta, 2003, 48, 1707.

4 F. Endres, ChemPhysChem, 2002, 3, 144.

5 Electrochemical Aspects of Ionic Liquids, ed. H. Ohno, John Wiley \& Sons, New York, 2005.

6 A. P. Abbott, G. Capper G, D. L. Davies and R. K. Rasheed, Chem.-Eur. J., 2004, 10(15), 3769-3774.

7 A. P. Abbott, D. Boothby, G. Capper, D. L. Davies and R. K. Rasheed, J. Am. Chem. Soc., 2004, 126, 9142-9147.

8 A. P. Abbott and K. J. McKenzie, Phys. Chem. Chem. Phys., 2006, 8(37), 4265-4279.

9 A. P. Abbott, G. Capper, D. Davies, R. K. Rasheed and V. Tambyrajah, Chem. Commun., 2003, 70-71.

10 A. P. Abbott, G. Capper, K. J. McKenzie and K. S. Ryder, Electrochim. Acta, 2006, 51, 4420.

11 A. P. Abbott, G. Capper, K. J. McKenzie, A. Glidle and K. S. Ryder, Phys. Chem. Chem. Phys., 2006, 8(36), 4214-4221.

12 A. R. Hillman, I. Efimov and M. Skompska, Faraday Discuss., 2002, 121, 423-439.

13 A. R. Hillman, A. Jackson and S. J. Martin, Anal. Chem., 2001, 73, 540.

14 A. R. Hillman, I. Efimov and S. K. Ryder, J. Am. Chem. Soc., 2005, 127, 16611.

15 (a) G. Sauerbrey, Z. Phys., 1959, 155, 206; (b) O. Schneider, A. Bund, A. Ispas, N. Borissenko, S. Z. El Abedin and F. Endres, J. Phys. Chem. B, 2005, 109(15), 7159-7168.

16 A. Bund, J. Solid State Electrochem., 2004, 8(3), 182-186.

17 S. Wehner, K. Wondraczek, D. Johannsmann and A. Bund, Langmuir, 2004, 20(6), 2356-2360. 